\title{
HELP@APP: development and evaluation of a self-help app for traumatized Syrian refugees in Germany - a study protocol of a randomized controlled trial
}

Johannes Golchert ${ }^{1 \dagger}$, Susanne Roehr ${ }^{1 * \dagger}$ (B), Franziska Berg ${ }^{1}$, Thomas Grochtdreis², Rahel Hoffmann³, Franziska Jung ${ }^{1}$, Michaela Nagl ${ }^{3}$, Anna Plexnies ${ }^{3}$, Anna Renner ${ }^{3}$, Hans-Helmut König ${ }^{2}$, Anette Kersting ${ }^{3+}$ and Steffi G. Riedel-Heller ${ }^{1+}$

\begin{abstract}
Background: Syrians represent the largest group among refugees in Germany. Many of them were exposed to sequential traumatizing events including war, escape and post-migration stressors, which significantly increase the risk to develop symptoms of posttraumatic stress and other mental disorders. However, there is a lack of adequate treatment options for traumatized refugees in Germany. Moreover, their access to psychosocial care is often restricted due to legal regulation, language barriers, and unclear cost coverage. We therefore aim to develop a lowthreshold supportive self-help app for Syrian refugees with posttraumatic stress symptoms. By conducting a randomized controlled trial, we further aim to evaluate the apps' efficacy, usability, acceptance, and economic health benefit/cost-effectiveness.

Methods: We will develop a modular, interactive self-help app in Arabic, which will be grounded on cognitivebehavioral models for the treatment of posttraumatic stress. Subsequently, screened positive (i.e., Syrian refugees, 18-65 years old, mild to moderate posttraumatic stress symptomatology as quantified by the Posttraumatic Stress Diagnostic Scale for DSM-5/PDS-5) participants (ideally up to $n=234$ ) will be randomly allocated to an intervention $(\mathrm{IG})$ and control group $(\mathrm{CG})$, respectively. Participants in the IG will gain access to the self-help app for one month, while participants in the CG will receive psychoeducational reading material in form of a comprehensive brochure on traumatization and posttraumatic stress. Measurements are scheduled before the intervention (T0), directly after the intervention (T1, one month later) and three months after the intervention (T2). Using linear mixed effect models, we will investigate change in posttraumatic symptomatology. We will also test for changes in secondary outcomes such as depression, anxiety, and quality of life. Moreover, we will inspect the usability and user acceptance of the app. To evaluate the app in terms of its economic health benefit, the incremental costeffectiveness ratio will be calculated.
\end{abstract}

Discussion: We plan to make the app freely available to the general public after evaluation. Thus, the app can help to add-on to routine care, which currently lacks sufficient and appropriate treatment options for Syrian refugees.

(Continued on next page)

\footnotetext{
* Correspondence: susanne.roehr@medizin.uni-leipzig.de

${ }^{\dagger}$ Johannes Golchert and Susanne Roehr contributed equally to this work.

Anette Kersting and Steffi G. Riedel-Heller shared last authorship.

${ }^{1}$ Institute of Social Medicine, Occupational Health and Public Health (ISAP),

Medical Faculty, University of Leipzig, Philipp-Rosenthal-Straße 55, 04103

Leipzig, Germany

Full list of author information is available at the end of the article
}

(c) The Author(s). 2019 Open Access This article is distributed under the terms of the Creative Commons Attribution 4.0 International License (http://creativecommons.org/licenses/by/4.0/), which permits unrestricted use, distribution, and reproduction in any medium, provided you give appropriate credit to the original author(s) and the source, provide a link to the Creative Commons license, and indicate if changes were made. The Creative Commons Public Domain Dedication waiver (http://creativecommons.org/publicdomain/zero/1.0/) applies to the data made available in this article, unless otherwise stated. 
(Continued from previous page)

Trial registration: German Clinical Trials Register/Deutsches Register Klinischer Studien (DRKS). Registration ID: DRKS00013782. Registered: 06th of July 2018.

Keywords: Posttraumatic stress, Trauma, Traumatization, Self-management program, App, mHealth, Digital health, Syria, Refugee, RCT, Intervention

\section{Background}

More than 5.6 million Syrians have fled their home country since the beginning of the Syrian civil war in 2011 [1]. Syrians represent the largest group among refugees in Germany. According to the Federal Office for Migration and Refugees [2], a total number of 158,657 Syrian refugees have applied for asylum in Germany in 2015. Compared to 2014, this corresponded to an increase of approximately $300 \%$. The upward trend continued through 2016, in which another 266,250 Syrians applied for asylum. Including the number of applications from 2017 (48,974) and 2018 (as of November: 41,345), this equals to a total number of 515,226 Syrian refugees in Germany between 2015 and November 2018.

Most of the Syrian refugees were typically exposed to a variety of potentially traumatizing situations. First, they might have been conflicted with wartime events in Syria, including bombardments and other combat operation. Second, the escape itself, which may last for months, sometimes even years (i.e., if they are aiming for European countries), may have caused existential threat, e.g. through dangerous crossings such as the Mediterranean Sea. Third, upon arrival at a host state, refugees are often confronted with so called post-migration stressors, such as discrimination and even physical violence [3]. Moreover, in Germany, refugees have to deal with time-consuming asylum procedures, prolonged waiting periods, and an ambiguous residence status, which can additionally increase the risk for developing psychiatric disorders [3, 4]. Formal hearings as part of the asylum procedure, in which the reasons for the escape are discussed, can be a further post-migration stressor. Indeed, current evidence suggests an increase in the development of intrusive experiences caused by asylum interviews as part of the asylum procedure [5]. Taken together, these events significantly increase the risk for developing trauma related and other disorders [6].

The most frequently reported disorders associated with war traumatization are posttraumatic stress disorder (PTSD) and major depression: studies reported prevalence rates of $33.5 \%$ for PTSD [7], and $29.5 \%$ for depression [8] among Syrian refugees. By contrast, in a representative study of the European general population that included six countries, the 12-month prevalence rates for PTSD and depression were 0.9 and 3.9\%, respectively [9]. PTSD is often accompanied by comorbid physical complaints, of which chronic pain is most common. Among refugees with PTSD, prevalence rates of clinically relevant chronic pain ranged from $76 \%$ [10] to $88 \%$ [11].

Recent PTSD guidelines by the German Association of the Scientific Medical Societies (AWMF) indicate that a trauma adaptive psychotherapy should be provided for every patient diagnosed with PTSD [12]. During the first 15 months after arrival to Germany, however, psychosocial care for refugees cannot be fully ensured due to initially restricted access to the health care system, which is regulated through specific asylum law (Asylbewerberleistungsgesetz/AsylbLG). By contrast, refugees who have stayed in Germany for more than 15 months gain access to the statutory health insurance that covers psychotherapy. According to a verdict of the Federal Social Court, however, health insurance funds are not obliged to cover the costs for a qualified translator, if needed. Instead, regional/local social security offices make individual decisions whether translator expenses can be refunded. However, refunding cannot be guaranteed, and it can take an enormous amount of extra time until a decision is made [3]. Moreover, the number of specialized psychotherapists and translators is limited. It is thus difficult to provide treatment for PTSD to all refugees in need. Indeed, there is a clear lack of adequate health services in this regard. Likewise, research has shown that traumatized individuals perceive additional barriers (e.g. concerns related to stigma, shame and rejection), which may hinder them to seek for adequate treatment [13].

Computerized/web-based and smartphone-based (apps) self-management programs that address posttraumatic stress in refugees are thus of particular importance as they a) may serve as a first supportive intervention and add-on to routine care, b) could fill a treatment gap in Germany, and c) may lower the threshold for refugees to seek help. Compared to face-to-face interventions, such digital approaches offer anonymity, which can help individuals to take up a respective treatment, who would otherwise maybe refuse to do so, e.g. due to concerns about stigmatization or feelings of shame [14, 15]. With the rapid increase of mobile phone use in recent years, apps for health care have also been developed. Since 
smartphones serve as an important communication tool and sometimes represent the only possibility for refugees to have access to the internet [16], smartphones might be of particular value in terms of health-related self-care programs in this particular group. As opposed to web-based approaches, mobile health (mHealth)-apps can be used flexibly and independently of internet access. Therefore, they are almost available without restrictions. In addition, mHealth-apps allow real-time monitoring of symptoms and behavior. Moreover, due to the easy and constant accessibility, they might improve treatment adherence [17]. Evidence for the efficacy of mHealth-apps has been reported for depression, anxiety, stress, and substance abuse [17, 18]. Likewise, a smartphone-based app for posttraumatic stress has proven to be efficient in veterans and in a general adult sample [19]. Two recent meta-analyses of internet-based interventions for posttraumatic stress reported medium to large effect-sizes with respect to a decrease in symptom severity $[20,21]$. Thus, such interventions have proven to be effective [22].

\section{Objectives}

Taken together, Syrian refugees are exposed to a variety of traumatizing situations making them especially vulnerable to posttraumatic stress symptoms. In Germany, psychosocial/psychotherapeutic care for refugees, however, is restricted by legal regulations and often unclear cost coverage. Moreover, there is a lack of easily accessible language and culture-sensitive interventions for posttraumatic stress in refugees. Therefore, we aim to develop an app-based self-management program in Arabic language for traumatized Syrian refugees in Germany. We further aim to evaluate the apps' efficacy and cost-effectiveness by conducting a randomized controlled trial (RCT) with two arms. Participants in the intervention group (IG) will gain access to the self-help app. Participants in the control group (CG) will be provided comprehensive psychoeducational reading material regarding PTSD. Using linear mixed effect models, we will investigate change in posttraumatic symptom load and related outcomes, such as depression, anxiety, and quality of life. App usability and user acceptance will additionally be assessed. To evaluate the app in terms of its health economic benefits, the incremental cost-effectiveness ratio (ICER) will be calculated.

\section{Expected benefits and harms}

We expect that the low-threshold app-based intervention will lower posttraumatic symptom severity and associated secondary outcomes in favor of overall improved wellbeing and quality of life. Furthermore, since our self-help app will be in Arabic language, basal information regarding treatment options and disease management can be provided to a broad group of traumatized refugees without causing costs due to translation. This can help to compensate for the lack of adequate psychotherapeutic care for Syrian refugees in Germany. In case of an unexpected increase in symptom severity - the trauma-related content might initially trigger unpleasant memories and feelings - both study groups will be provided with contact information for professional help in case of emergency. Moreover, the occurrence of negative effects, regardless whether or not related to the trial, will be monitored.

\section{Methods}

We aim to develop and evaluate a self-help app for traumatized Syrian refugees. This study protocol describes both steps in detail in adherence to the SPIRIT 2013 statement (Standard Protocol Items: Recommendations for Interventional Trials) [23].

\section{Development of the self-help app Theoretical and clinical framework}

Our interactive self-help app will rely on (i) evidence-based cognitive-behavioral therapy (CBT) for PTSD [24], and (ii) evaluated internet-based self-care approaches for the treatment of posttraumatic stress $[25,26]$. Critically, meeting the specific needs of refugees as well as adapting the content in a culturally sensitive manner will be key to the development of our self-help app. Initially, interviews with approximately 510 target persons will thus be carried out to assess culturally sensitive aspects (e.g. different concepts of disease and disease management), that may be important for the intervention. To ensure full functionality and usability of the app, representatives of the target group will accompany all steps of the developmental process through regular consultation.

\section{Structure and content of the self-help app}

As can be seen in Fig. 1, the app will be set up modularly. Initially, participants will be guided through general user instructions and information about the association between war, escape, and posttraumatic stress will be provided ("onboarding"). Then, participants will be able to flexibly work through two modules ("trauma" and "resources"), which will consist of several submodules.

The "trauma"-submodules will address consequences of traumatization, especially focusing on posttraumatic stress symptoms as defined by the Diagnostic and Statistical Manual of Mental Disorders, 5th Edition (DSM-5) [27]. In addition, symptoms of depression and anxiety will be introduced and explained. All submodules will provide (i) psychoeducational information (e.g. what are intrusions and flashbacks, what is somatization), and (ii) basic self-help techniques and skills with respect to symptom management (e.g. positive and negative coping strategies, cognitive restructuring, emotion regulation). We will also implement a self-test (PTSD Checklist for DSM-5/PCL-5) [28], that can 


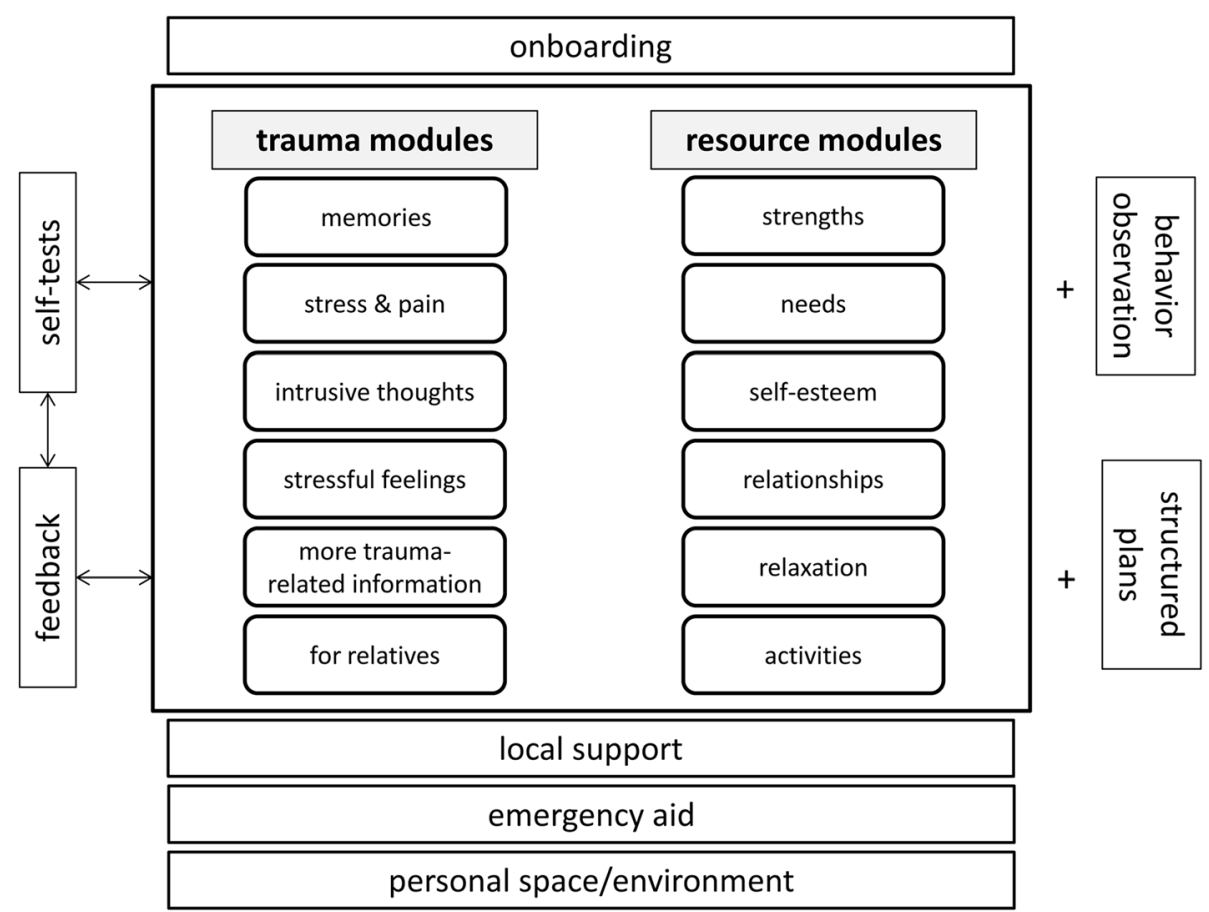

Fig. 1 Study design for a randomized controlled trial to evaluate a self-help app for traumatized Syrian refugees living in Germany

be completed at any time in order to monitor symptom severity and to provide automated individually tailored feedback regarding progress and potential problems.

The "resources"-submodules will impart (i) psychoeducational information (e.g. about physical and mental needs, consequences of experienced traumata on self-esteem and relationships) as well as (ii) techniques and skills to strengthen and support own resources and self-care (e.g. setting goals, planning pleasant activities, gaining social support, relaxation techniques, mindfulness).

\section{Usability}

To maximize usability, content related materials will be provided in Arabic. Moreover, the app will additionally be complemented by video and audio sequences as well as interactive games and exercises. All submodules will be available at all times, giving users the possibility to flexibly switch between sections depending on their personal needs. The app will also provide a clearly visible emergency button with contact information regarding professional help in case of emergency. The app will be usable offline.

With respect to the technical implementation, the app development will take place in cooperation with the e-mental health program provider "Frühlingsproduktionen".

\section{Evaluation of the self-help app Participants}

Study participants will be Syrian refugees living in the urban area of Leipzig, Germany, who will be recruited using a multi-strategic approach [29]. If contacted refugees are willing to participate, written informed consent will be obtained. Trained native Arabic speaking study nurses will screen up to $n=1200$ refugees for eligibility defined by the following inclusion and exclusion criteria, respectively.

\section{Inclusion criteria:}

- Syrian refugee living in Leipzig, Germany, aged 18-65 years

- experience of at least one traumatic event and subsequent mild to moderate posttraumatic stress symptom severity (Posttraumatic Diagnostic Scale for DSM-5/PDS-5 = 11-59 [30])

- owning a compatible device (Android / iOS)

\section{Exclusion criteria:}

- severe posttraumatic stress symptomatology (PDS$5 \geq 60$ [30])

- severe depressive symptomatology (Patient Health Questionnaire/PHQ-9 $\geq 20$ [31])

- acute suicidal tendencies (Depressive Symptom Inventory-Suicidality Subscale/DSI-SS $\geq 3$ [32])

- current psychotherapy/psychiatric treatment and/or psychopharmaceutical medication

- pregnancy

\section{Intervention}

The apps' efficacy and usability as well as the overall associated cost effectiveness of the intervention will be 
evaluated in a prospective RCT with an IG and a CG (see Fig. 2). Following the initial screening and study group assignment, participants in the IG will gain access to the self-help app via person-specific log-in data (to avoid group contamination) for one month. During that time, the CG will obtain psychoeducational reading material about traumatization and posttraumatic stress (identical to the information delivered by the app) in form of a comprehensive brochure in Arabic.

\section{Study adherence}

Prior to randomization, participants will be pointed towards the required investment of time and effort necessary to complete the study in order to lower the risk for dropout.

Treatment adherence of the IG will be objectively assessed through the apps' meta-data log-files that store information about the number of login-times and worked-through modules. Likewise, dropout assessment will be conducted to understand reasons for non-adherence.

Participants of both groups are assured to contact the study nurses in case of arising problems regarding current symptom burden, for example. Similar to the apps' emergency button, the psychoeducational reading material will clearly state contact information regarding professional help in case of emergency.

\section{Outcomes}

Table 1 gives an overview of all outcomes and associated measures to be implemented throughout the entire study. Instruments that are not available in Arabic language will be translated according to recommendations of the "European Social Survey Programme" and "Cross-Cultural Survey Guidelines" respectively [33, 34]. Based on the TRAPD (Translation, Review, Adjudication, Pretesting, and Documentation) model, two independent and external translators will each provide drafts of a common forward and backward translation, which will subsequently be discussed and reviewed by an expanded group of bilingual experts. The approved versions will then be pretested. The entire procedure will be repeated, if necessary. This multi-step team translation approach not only ensures the reliable detection and elimination of problems of equivalence between the different questionnaire versions [35], but also takes into account the cultural specifics of the respective language. All other study materials (e.g. informed consent, brochure for the CG) will be translated by bilingual experts as well.

We hypothesize that the app intervention reduces posttraumatic stress symptomatology (primary outcome), quantified using the Posttraumatic Diagnostic Scale for DSM-5 (PDS-5 [30]). The PDS-5 is a well-established self-report measure based on the diagnostic criteria of the DSM-5, which allows the assessment of posttraumatic stress symptom severity.

Regarding secondary outcomes, we moreover expect the intervention to reduce symptoms of depression, anxiety, and somatization. Positive intervention-related effects are also expected regarding quality of life, self-efficacy,

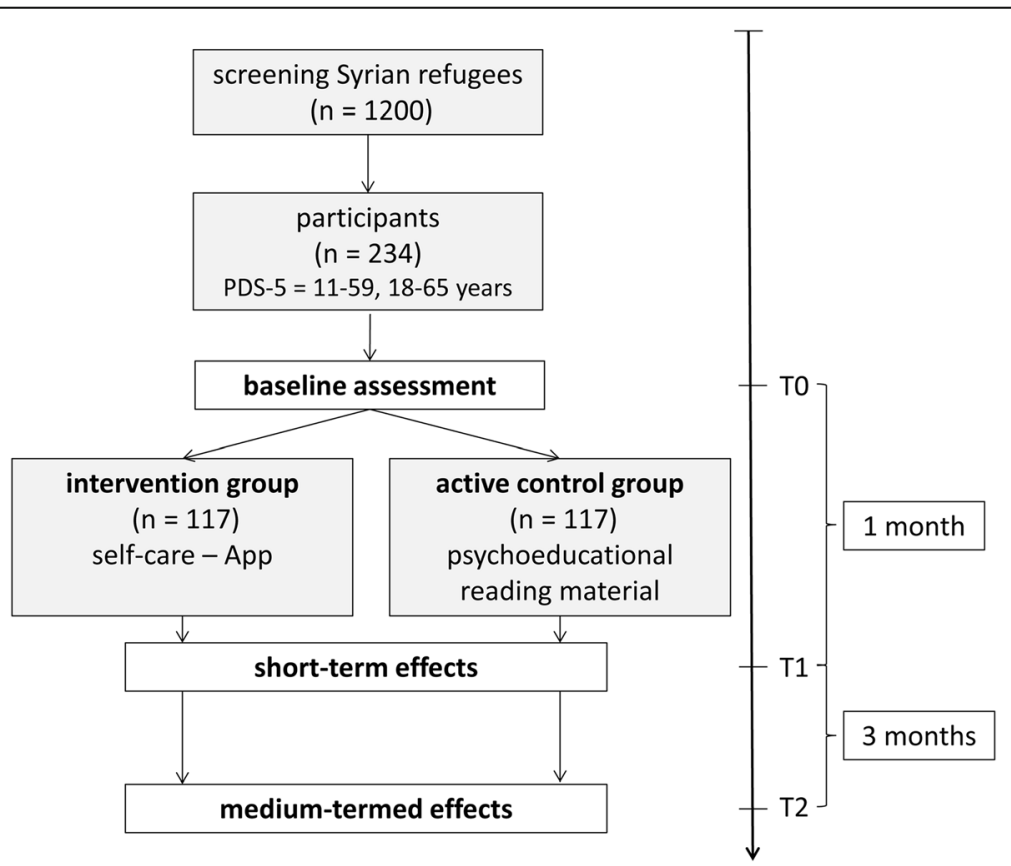

Fig. 2 Overview of the structure and contents of a self-help app for traumatized Syrian refugees living in Germany 
Table 1 Overview of data to be collected for the evaluation of a self-help app for traumatized Syrian refugees

\begin{tabular}{|c|c|c|c|c|}
\hline Construct (Assessment) & Screening & Baseline (T0) & Follow-up (T1 / T2) & Arabic translation necessary \\
\hline \multicolumn{5}{|l|}{ Sociodemographics } \\
\hline Age & $x$ & & & yes \\
\hline Sex & $x$ & & & yes \\
\hline Education & $x$ & & & yes \\
\hline Marital status & $x$ & & & yes \\
\hline Living situation & $x$ & & & yes \\
\hline Residence status & $x$ & & & yes \\
\hline Occupational status & $x$ & & & yes \\
\hline Monthly income & $x$ & & & yes \\
\hline \multicolumn{5}{|l|}{ Escape-related information } \\
\hline Home country & $x$ & & & yes \\
\hline Date of escapes' beginning & $x$ & & & yes \\
\hline Duration of stay in Germany & $x$ & & & yes \\
\hline Means of escape & $x$ & & & yes \\
\hline Strength of bonding to homeland / current residence & $x$ & & & yes \\
\hline Future plans & $x$ & & & yes \\
\hline Satisfaction with current situation & $x$ & & & yes \\
\hline \multicolumn{5}{|l|}{ Additional inclusion criteria } \\
\hline $\begin{array}{l}\text { Smartphone availability, no current psychiatric treatment and/or no } \\
\text { psychopharmaceutic medication, no pregnancy }\end{array}$ & $x$ & & & yes \\
\hline Suicidal tendencies (DSI-SS) & $x$ & $x$ & $x$ & yes \\
\hline \multicolumn{5}{|l|}{ Primary Outcome } \\
\hline Post-traumatic stress disorder (PDS - 5) & $x$ & $x$ & $x$ & yes \\
\hline \multicolumn{5}{|l|}{ Secondary Outcomes } \\
\hline Depression (PHQ-9) & $x$ & $x$ & $x$ & no \\
\hline Anxiety (GAD-7) & & $x$ & $x$ & no \\
\hline Somatization (PHQ-15) & & $x$ & $x$ & no \\
\hline Quality of life (EQ-5D-5 L) & & $x$ & $x$ & no \\
\hline General self-efficacy (GSE) & & $x$ & $x$ & yes \\
\hline Self-stigmatization (SSMIS-SF) & & $x$ & $x$ & yes \\
\hline Ambiguous loss and prolonged grief & & $x$ & $x$ & yes \\
\hline Social network (LSNS) & & $x$ & $x$ & yes \\
\hline Social support (ESSI) & & $x$ & $x$ & yes \\
\hline Post-traumatic growth (PGI) & & $x$ & $x$ & yes \\
\hline Resilience (RS-13) & $x$ & & & yes \\
\hline Religious beliefs (Z-7) & & $x$ & $x$ & yes \\
\hline Healthcare resource use & & $x$ & only T2 & yes \\
\hline App usability (SUS) $)^{a}$ & & $x$ & only T1 & yes \\
\hline User acceptance (TAM 3) ${ }^{a}$ & & $x$ & only T1 & yes \\
\hline
\end{tabular}

Note: ${ }^{a}$ to be assessed only in the intervention group

DSI-SS Depressive Symptomatology Index - Suicidality Scale [47], PDS-5 Posttraumatic Diagnostic Scale for DSM-5 [30], PHQ-9 Patient Health Questionnaire [31], GAD General Anxiety Disorder [48], EQ-5D-5L Euroqol Five Dimensions Questionnaire [49], GSE General Self-efficacy [50], SSMIS-SF Self-stigma of Mental IIIness Scale - Short Form [51], LSNS Lubben Social Network Scale [52], ESSI Enriched Social Support Inventory [53], PGI Posttraumatic Growth Inventory [54], RS-13 Resilience Scale [55], Z-7 Zentralitätsskala [56], SUS System Usability Scale [36], TAM-3 Technology Acceptance Model [37]

Ambiguous loss and prolonged grief - questions have been adapted from the German version of the Complicated Grief Inventory (ICG-D; [57]); Healthcare resource use - questionnaire was adapted from previous investigations and is available from the authors upon request [58] 
perceived stigmatization, social support, ambiguous loss, and posttraumatic growth.

Use of resources in terms of health demands will be assessed using a cost book (bottom-up approach). Furthermore, we aim to gather information on user acceptance and usability of the self-help app using the System Usability Scale (SUS) [36] and Technology Acceptance Model (TAM3 [37]), respectively.

Last, meta-data stored in the app's log-files will provide quantitative information about the use of the app, e.g. number of days used, number of worked-through modules, or operating time.

\section{Timeline}

After positive screening, study participants will be enrolled. Measurements are scheduled directly before the intervention (baseline, T0/pre), immediately after the intervention (T1/post, one month later), and three month after the intervention (T2/follow-up) to test for medium-termed treatment effects. A detailed overview of the schedule of enrolment, interventions and assessments is given in Fig. 3 .

\section{Sample size}

Anticipated sample size was calculated based on recent research evaluating the efficacy of telemedical-based treatment of posttraumatic stress symptoms [20, 21, 38]. Given a moderate between-group effect at T1 (Cohen's $d=0.5$ ), a significance level of $\alpha=0.05$ and a statistical power of $1-\beta=0.90$, optimal sample size for estimating a significant treatment effect is $n=140$. Expecting a drop-out rate of approximately $40 \%$ (e.g. due to change in residence status), $n=94$ participants have to be included additionally, yielding an ideal baseline sample of $n=234$ participants at T0 [39].

\section{Recruitment}

A multi-strategic approach to recruit Syrian refugees living in the urban area of Leipzig, German, will be targeted. This includes using established contacts as well as building new contacts with facilities frequented by Syrian refugees (e.g. refugee related clubs, associations and organizations, psychosocial centers, information centers, language schools, community colleges, universities, registration authorities, hospitals, clinics, primary care practices, psychotherapeutic practices, etc.). These facilities act as multipliers to reach out to potential study participants. Likewise, participants will be recruited from local reception centers and other forms of collective accommodation centers. We will use social media, instant messaging and online tools to spread information about the study. The local media will be involved. Bilingual flyers and posters will be displayed all across the city in appropriate public spots. During the recruitment phase, we will have an information booth at local events that might attract Syrian refugees. Importantly, since Syrian refugees are well connected with each other, we aim to reach additional participants of the Syrian community by using a snowball sampling approach, e.g. we ask participants to spread information about the study within their personal social network [29].

\section{Allocation and randomization}

Participants identified as eligible during screening will subsequently be randomly assigned to the IG or CG. Group assignment will follow a 1:1 ratio utilizing randomized permuted blocks of six, stratified by age and sex. This procedure ensures both balance in sample size across groups and control of important covariates. An external, independent statistician will generate the randomization block lists with a respective computer program ("blockrand"-package [40] written for R [41]). The different lists will be coded by this person so that the study coordinator, responsible for individual group allocation, remains blind to the lists' strata identity.

\section{Blinding}

Besides randomization, the data analysts, who will conduct the primary analysis concerning the hypothesized group differences (IG vs. CG) in our primary and secondary outcome measures (see above), will be blind to group assignment.

\section{Data collection}

All assessments will be carried out in form of structured face-to-face interviews led by trained native Arabic speaking study nurses. The structured interviews will be present in form of paper-and-pencil assessments that the study nurses will complete to collect data on sociodemographics, primary and secondary outcomes using standardized questionnaires, as detailed above. Additionally, data will be collected to monitor possible negative effects and reasons for dropout (see below).

Data on the app usage will be collected through meta-data $\log$-files that store anonymous information about the number of login-times and worked-through modules.

\section{Data management}

Data entry will take place successively and timely after collection using a statistical package. However, a data completeness and consistency check will take place beforehand. In case of missing answers to items, reasons for missing information will be clarified and efforts undertaken to reach completion. De-identified data will be entered in a data mask which will be password-protected and stored locally, with access granted only to study personnel who have signed data protection wavers. Anonymous log-file data of 


\begin{tabular}{|c|c|c|c|c|c|c|c|c|}
\hline \multirow[b]{3}{*}{ TIMEPOINT** } & \multicolumn{8}{|c|}{ STUDY PERIOD } \\
\hline & \multirow{2}{*}{$\frac{\text { Enrolment }}{-t_{1}}$} & \multirow{2}{*}{$\frac{\text { Allocation }}{0}$} & \multicolumn{5}{|c|}{ Post-allocation } & \multirow{2}{*}{$\frac{\text { Close-out }}{t_{3}}$} \\
\hline & & & $t_{1}$ & $t_{2}$ & $t_{3}$ & $t_{4}$ & etc. & \\
\hline \multicolumn{9}{|l|}{ ENROLMENT: } \\
\hline \multirow{2}{*}{$\begin{array}{l}\text { Informed consent } \\
\text { Eligibility screen }\end{array}$} & $x$ & & & & & & & \\
\hline & $x$ & & & & & & & \\
\hline PTSD diagnosis & $x$ & & & & & & & \\
\hline Allocation & & $x$ & & & & & & \\
\hline \multicolumn{9}{|l|}{ INTERVENTIONS: } \\
\hline \multicolumn{9}{|l|}{$\begin{array}{r}\text { Syrian refugees } \\
\text { (self-help app) }\end{array}$} \\
\hline \multicolumn{9}{|l|}{$\begin{array}{c}\text { Syrian refugees } \\
\text { (control group) }\end{array}$} \\
\hline \multicolumn{9}{|l|}{ ASSESSMENTS: } \\
\hline \multicolumn{9}{|l|}{$\begin{array}{r}\text { Sociodemographics } \\
\text { Inclusion criteria } \\
R S-13\end{array}$} \\
\hline $\begin{array}{r}P D S-5 \\
P H Q-9 \\
D S I-S S \\
\text { GAD-7 } \\
P H Q-15 \\
\text { EQ-5D-5L } \\
\text { GSE } \\
\text { SSMIS-SF } \\
\text { Amb. Ioss / grief } \\
\text { LSNS } \\
\text { ESSI } \\
P G I \\
Z-7\end{array}$ & & & $x$ & $x$ & $x$ & & & $x$ \\
\hline $\begin{array}{l}\text { Health care } \\
\text { resource use }\end{array}$ & & & $x$ & & $X$ & & & $x$ \\
\hline $\begin{array}{l}\text { SUS* } \\
\text { TAM3* }\end{array}$ & & & $x$ & $x$ & & & & \\
\hline
\end{tabular}

Fig. 3 Schedule of enrollment, interventions and assessments. Completed SPIRIT 2013 figure of recommended content of enrolment, interventions, and assessments. Abbreviations: $\mathrm{t} 1$ = baseline; $\mathrm{t} 2=4$ weeks after baseline (post intervention); $\mathrm{t} 3=16$ weeks after baseline (follow-up). *assessed in intervention group only

app usage will be collected, stored and transferred using SSL-technology to ensure data encryption and protection. In fact, specific arrangements regarding data protection (e.g. separation of evaluation and program, no real names for program login) will be defined by a separate data protection concept.

\section{Statistical analysis}

All acquired data will be examined with respect to potential inconsistencies and missing values, which will be replaced using multiple imputation methods [42], if appropriate. To detect potential systematic biases, a drop-out analysis will be performed. Internal consistencies of the newly translated inventories will be calculated to evaluate their reliability. All subsequent analyses will be treated as intention-to-treat or completer-analyses. Data-analysts will be blinded for group allocation.

Changes in primary and secondary outcomes will be described using mean scores and standard deviations, among others. Short and medium termed treatment effects will statistically be inferred by using linear mixed effect models accounting for relevant covariates. Usability as well as user acceptance of the app 
will be evaluated using descriptive statistics. The significance level of all analyses will be set to $p<0.05$.

To assess the potential health economic benefits, we will perform analyses of the (i) actual costs and (ii) the ICER of the intervention. Costs will be quantified by the frequency of how often physicians and therapists are consulted (including days spent in a hospital) and valuated using current standard costs [43]. The app development expenses will be treated as economically reasonable overhead costs and added accordingly. To assess the interventions' economic efficiency, the ICER will be calculated as the ratio between the difference of the actual costs and the difference of the averaged treatment effects (indexed by either quality-adjusted life years or primary outcome scores) between IG and CG. Respective confidence intervals will be derived by non-parametric bootstrapping. To visualize the ICERs statistical uncertainty, cost-effectiveness-acceptance curves will be constructed, indicating the probability of an acceptable ICER in relation to willingness to pay for an effect. Alternatively, net benefit regressions [44] can be applied to estimate the respective group specific net benefits while accounting for potentially confounding variables such as age, sex, and education.

The results of the study will be reported according to the guidelines of the Consolidated Standards of Reporting Trials (CONSORT) statement [45].

\section{Monitoring}

\section{Data monitoring}

A data monitoring committee (DMC) will not be established for several reasons. First, periodic inspection does not seem justified as trial duration is short (4 weeks). Second, overall risk associated with the self-help intervention is considered low, and therefore, the likelihood for the need to modify or discontinue the trial is considered insignificant. However, precautions have been taken to minimize the potential of any harm towards individual study participants, as detailed below.

\section{Harms}

Preventively, we will only enroll study participants without severe posttraumatic and/or depressive symptomatology and acute suicidality. The self-help program targets individuals with mild to moderate symptom severity. Despite these precautions, the occurrence of negative effects during the trial in individual cases cannot be entirely ruled out. Therefore, potential negative effects will be monitored through a standardized assessment procedure at follow-up 1 (post-intervention) and follow-up 2 (four months after baseline). Negative effects are defined as adverse events (AE) and severe adverse events (SAE). Specifically, AE comprise i) an increase in target symptoms (posttraumatic and depressive symptomatology, suicidality), ii) the occurrence of novel psychological or physical symptoms, and iii) any negative events, all of which may or may not be associated with trial participation, in both IG and CG. Participants will be questioned whether any $\mathrm{AE}$ is thought to emerge from trial participation. SAE are defined as severe negative events that occur during study participation and result in adverse reactions requiring some form of high intensity treatment (i.e., deliberate self-harm, suicide attempt, life-threatening events, non-elective or extended hospitalization, an event causing chronic or serve disability) or fatality, including suicide. SAE will be surveyed and reported regardless if they are deemed related to the trial or not. The principle investigator $(\mathrm{SRH})$ will be immediately informed about the occurrence of any $\mathrm{AE}$ and SAE. Likewise, a consensus conference (incl. the principle investigator and clinical investigators) will be held immediately to decide on appropriate measures in response to $\mathrm{AE}$ and SAE. Moreover, data collected on $\mathrm{AE}$ and SAE will be analyzed and reported as part of the study outcomes.

Practically, during the trial, study participants of both groups (IG and CG) will be provided with detailed information regarding emergency hotlines and professional help, if needed, i.e., in case of unexpected symptom deterioration. The IG will have access to this information via an emergency button leading to a help menu within the app. The CG receives the same information as part of the psychoeducational reading material that is handed out at baseline. In addition, study participants of both groups will be encouraged to contact our study nurses if they need further support to find emergency help.

\section{Auditing}

Auditing will take place in form of independent reviews of the data collection across all assessment waves. Specifically, $5 \%$ of the questionnaires at baseline, follow-up 1 and follow-up 2, respectively, will be randomly drawn and inspected regarding their degree of matching with the database input. Source data verification will be performed independently from investigators and study sponsors by commissioned, external statisticians.

\section{Protocol amendments}

In case of necessary protocol amendments, we will describe all relevant details/changes and associated rationales.

\section{Dissemination policy}

The results of the RCT will be published in international, peer-reviewed journals. Moreover, we plan to present our findings at international and national conferences and meetings relevant to the field. To reach an even broader publicity, we aim to cooperate with the largest health insurance provider in Germany (AOK - Federal Association of Local Health Insurance Funds), national institutions 
concerned with migration and integration as well as stakeholders/protagonists of refugee care centers. We will additionally arrange several information events for experts from the field of psychiatry and psychology as well as for trauma-specific experts and representatives of psychosocial counselling centers. Lastly, we aim to share our results via public media channels. We aim to make the app freely available to everyone.

\section{Discussion}

We aim to develop and evaluate an interactive self-help app in Arabic for Syrian refugees with symptoms of posttraumatic stress. We expect that the use of our self-help app will lower respective and associated comorbid symptoms, e.g. depression or anxiety, over a course of four weeks. Moreover, our evaluation will consider the intervention's cost-effectiveness as well as aspects regarding usability and user acceptance. We plan to make the app publicly available. Hence, we hope to provide a flexible low-threshold treatment for Syrian refugees, who suffer from the consequences of traumatization, but who may lack access to an appropriate treatment. If proven to be efficient, the self-help app could fill an important treatment gap in health services provision to a large group in need, and thus, could significantly improve the psychosocial care for Syrian refugees in Germany. Moreover, after implementation in public health, applicability may not only be restricted to Syrian refugees, but the app can be helpful for refugees from other Arabic-speaking countries with similar cultural backgrounds.

However, the realization of the project might be subject to some risks that have to be considered. Regarding the app development, it is important to adequately adapt the content to culture-sensitive aspects (e.g. disease concepts, the specific situation of refugees in Germany). Therefore, insights obtained from interviews with respective focus groups will be integrated. While the translation of the app materials and research inventories need to be culturally sensitive, it should not lose the necessary precision. In order to optimally balance these demands, we will cooperate with qualified translators, bilingual and native Arabic speaking experts, who will translate and review all materials within the standard forward and backward translation process (TRAPD-Model). Lastly, difficulties might occur with respect to recruitment and adherence of participants, e.g. due to contact barriers, dropout, or imminent deportation. We therefore aim for a multi-strategic recruitment approach. In addition, we applied a high dropout rate (40\%) for sample size calculation.

Although a recent meta-analysis demonstrated the efficacy of internet-based interventions for posttraumatic stress $[20,21]$, the associated advantages and potentials remain unused in Germany. The same seems to be true with respect to mHealth approaches. Although, there are plenty of self-help apps available, most of them have not been validated yet [46], and might not meet the specific needs of traumatized Syrian refugees. Thus, we will develop and evaluate an interactive self-help app in Arabic language, which will prioritize the special needs of Syrian refugees in Germany. We expect that our results will extend current knowledge regarding the efficacy of mobile health interventions in the field of psychosocial care for traumatized Syrian refugees in Germany. The overall goal is to provide the app to the general public for free as a low-threshold add-on to routine care, which currently lacks adequate treatment options for refugees with posttraumatic stress.

\section{Abbreviations}

AE: Adverse event; App: Application; AWMF: German Association of the Scientific Medical Societies; CBT: Cognitive-behavioral therapy; CG: Control group; DMC: Data monitoring committee; DSM-5: Diagnostic and Statistical Manual of Mental Disorders, 5th Edition; ICER: Incremental cost-effectiveness ratio; IG: Intervention group; mHealth: Mobile health; PDS-5: Posttraumatic

Diagnostic Scale for DSM-5; PHQ-9: Patient Health Questionnaire;

PTSD: Posttraumatic stress disorder; RCT: Randomized controlled trial; SAE: Severe adverse event

\section{Acknowledgements}

We like to thank in advance all participants and collaborators. Members of the HELP@APP Study Group: Steffi G. Riedel-Heller (Principle Investigator), Anette Kersting, Hans-Helmut König (Co-Principal Investigators); Maram Alhamarneh, Zain Assaad, Ismail Ayoub, Franziska Berg, Judith Dams, Johannes Golchert, Thomas Grochtdreis, Rahel Hoffmann, Franziska Jung, Yamen Khamis, Michaela Nagl, Anne Plexnies, Anna Renner, Susanne Röhr.

\section{Funding}

The study is funded by the Innovationsfonds (01VSF16033) of the Federal Joint Committee (Gemeinsamer Bundesausschuss/G-BA). The study has undergone full external peer reviews as part of the funding process and the funding body had no other role with respect to the design of the study and in writing the study protocol. They will have no ultimate authority regarding data collection, management, analysis, interpretation of the data and writing the reports/publications

We acknowledge support from the German Research Foundation (DFG) and Universität Leipzig within the program of Open Access Publishing.

\section{Availability of data and materials}

Access to the final dataset will initially be limited to the study contributors. We plan to make participant-level data and statistical code available to other researchers upon request and only after signing a non-disclosure agreement. The full study protocol will be made freely available through open access publication.

\section{Organizational structure and responsibilities}

Development and evaluation of the interactive self-help app is realized through cooperation between the Institute of Social Medicine, Occupational Medicine and Public Health (ISAP), Medical Faculty, University of Leipzig; the Department of Psychosomatic Medicine and Psychotherapy of the University of Leipzig, and the Department of Health Economics and Health Services Research at the University Medical Center Hamburg-Eppendorf. Specifically, the development of the app will mainly be conducted at the Department of Psychosomatic Medicine and Psychotherapy of the University of Leipzig. With respect to evaluation, the ISAP is specifically responsible for study preparation, participant recruitment, conduction of the trial, documentation as well data analysis and interpretation. Cost-effectiveness analyses will be conducted by the Department of Health Economics and Health Services Research at the University Medical Center Hamburg-Eppendorf. 


\section{Authors' contributions}

$\mathrm{SRH}, \mathrm{AK}$ and $\mathrm{HHK}$ conceived and designed the study and obtained funding for the project. JG and SR wrote the manuscript. SRH, AK, HHK, FB, TG, RH, FJ, $M N, A P, A R$ and $H H K$ revised the manuscript for important intellectual content. All authors read and approved the final version of the manuscript. Authorship eligibility guidelines for upcoming reports of the trial's results are in place, professional writers are not intended to be employed at any time.

\section{Ethics approval and consent to participate}

This study was approved by the Ethics committee of the Medical Faculty of the University of Leipzig, Germany (ID: 111-17-ek) and is conducted in accordance with the Declaration of Helsinki and the ICH guidelines for Good Clinical Practice (GCP). All participants will be informed verbally and in written about the aims and practical implementation of the study. This also includes a clarification about data security according to latest legal standards. Participants' inclusion will only be allowed after agreement to the written informed consent. Participation is completely optional and can be terminated at any point without consequences.

\section{Consent for publication}

Written informed consent for publication of study results based on the deidentified data collected from participants during trial participation is obtained upon enrollment.

\section{Competing interests}

The authors declare that they have no competing interests.

\section{Publisher's Note}

Springer Nature remains neutral with regard to jurisdictional claims in published maps and institutional affiliations.

\begin{abstract}
Author details
${ }^{1}$ Institute of Social Medicine, Occupational Health and Public Health (ISAP), Medical Faculty, University of Leipzig, Philipp-Rosenthal-Straße 55, 04103 Leipzig, Germany. ${ }^{2}$ Department of Health Economics and Health Services Research, Hamburg Center for Health Economics, University Medical Center Hamburg-Eppendorf, Hamburg, Germany. ${ }^{3}$ Department of Psychosomatic Medicine and Psychotherapy, University Medical Center Leipzig, Leipzig, Germany.
\end{abstract}

\section{Received: 25 January 2019 Accepted: 10 April 2019} Published online: 30 April 2019

\section{References}

1. UNHCR. UNHCR. 2018. http://www.unhcr.org/syria-emergency.html. Accessed 15 May 2018.

2. Bundesamt für Migration und Flüchtlinge. Aktuelle Zahlen zu Asyl. 2018. http://www.bamf.de/DE/Infothek/Statistiken/Asylzahlen/AktuelleZahlen/ aktuelle-zahlen-asyl-node.html. Accessed 15 May 2015.

3. Böttche M, Heeke C, Knaevelsrud C. Sequenzielle Traumatisierungen, Traumafolgestörungen und psychotherapeutische Behandlungsansätze bei kriegstraumatisierten erwachsenen Flüchtlingen in Deutschland. Bundesgesundheitsblatt Gesundheitsforschung Gesundheitsschutz. 2016;59: 621-6. https://doi.org/10.1007/s00103-016-2337-4.

4. Laban CJ, Gernaat HB, Komproe HH, Schreuders BA, De Jong JT. Impact of a long asylum procedure on the prevalence of psychiatric disorders in Iraqi asylum seekers in The Netherlands. J Nerv Ment Dis. 2004;192:843-51. https://doi.org/10.1097/01.nmd.0000146739.26187.15.

5. Schock K, Rosner R, Knaevelsrud C. Impact of asylum interviews on the mental health of traumatized asylum seekers. Eur J Psychotraumatol. 2015;6: 26286.

6. Assion HJ, Bender $\mathrm{M}$, Koch E, Pollmächer T. Flüchtlinge in Not Fachverbändezbd Kliniken sind gefordert: Psychiatrische und psychotherapeutische Hilfe frühzeitig anbieten. Psychiatr Prax. 2016;43:456. https://doi.org/10.1055/s-0042-119811.

7. Alpak G, Unal A, Bulbul F, Sagaltici E, Bez Y, Altindag A, et al. Post-traumatic stress disorder among Syrian refugees in Turkey: a cross-sectional study. Int J Psychiatry Clin Pract. 2015;19:45-50. https://doi.org/10.3109/13651501. 2014.961930.
8. Gammouh OS, Al-Smadi AM, Tawalbeh LI, Khoury LS. Chronic diseases, lack of medications, and depression among Syrian refugees in Jordan, 20132014. Prev Chronic Dis. 2015;12:E10. https://doi.org/10.5888/pcd12.140424.

9. Valderas JM, Kotzeva A, Espallargues M, Guyatt G, Ferrans CE, Halyard MY, et al. The impact of measuring patient-reported outcomes in clinical practice: a systematic review of the literature. Qual Life Res. 2008;17:179-93. https:// doi.org/10.1007/s11136-007-9295-0.

10. Dahl S, Dahl C-I, Sandvik L, Hauff E. Chronic pain in traumatized refugees. Tidsskr Den Nor Laegeforening Tidsskr Prakt Med Ny Raekke. 2006;126:60810.

11. Teodorescu D, Heir T, Siqveland J, Hauff E, Wentzel-Larsen T, Lien L. Chronic pain in multi-traumatized outpatients with a refugee background resettled in Norway: a cross-sectional study. BMC Psychol. 2015;3:7. https://doi.org/10. 1186/s40359-015-0064-5

12. Flatten, G., Gast, U., Hofmann, A. et al. S3-Leitlinie Posttraumatische Belastungsstörung ICD-10: F43.1. Trauma Gewalt. 2011;5:202-10.

13. Kantor V, Knefel M, Lueger-Schuster B. Perceived barriers and facilitators of mental health service utilization in adult trauma survivors: a systematic review. Clin Psychol Rev. 2017;52:52-68. https://doi.org/10.1016/j.cpr.2016.12 001.

14. Musiat P, Tarrier N. Collateral outcomes in e-mental health: a systematic review of the evidence for added benefits of computerized cognitive behavior therapy interventions for mental health. Psychol Med. 2014;44: 3137-50. https://doi.org/10.1017/S0033291714000245.

15. Kersting A, Schlicht S, Kroker K. Internettherapie. Möglichkeiten und Grenzen. Nervenarzt. 2009;80:797-804. https://doi.org/10.1007/s00115-0092721-5.

16. Maitland C, Xu Y. A social informatics analysis of refugee Mobile phone use: a case study of Za'atari Syrian refugee camp. SSRN Electron. 2015.

17. Donker T, Petrie K, Proudfoot J, Clarke J, Birch M, Christensen H. Smartphones for smarter delivery of mental health programs: a systematic review. J Med Internet Res. 2013;15:e247. https://doi.org/10.2196/jmir.2791.

18. Harrison V, Proudfoot J, Wee PP, Parker G, Pavlovic DH, Manicavasagar V. Mobile mental health: review of the emerging field and proof of concept study. J Ment Health. 2011;20:509-24. https://doi.org/10.3109/09638237. 2011.608746.

19. Kuhn E, Kanuri N, Hoffman JE, Garvert DW, Ruzek Jl, Taylor CB. A randomized controlled trial of a smartphone app for posttraumatic stress disorder symptoms. J Consult Clin Psychol. 2017;85:267-73.

20. Kuester A, Niemeyer H, Knaevelsrud C. Internet-based interventions for posttraumatic stress: a meta-analysis of randomized controlled trials. Clin Psychol Rev. 2016;43:1-16. https://doi.org/10.1016/j.cpr.2015.11.004.

21. Sloan DM, Gallagher MW, Feinstein BA, Lee DJ, Pruneau GM. Efficacy of telehealth treatments for posttraumatic stress-related symptoms: a metaanalysis. Cogn Behav Ther. 2011;40:111-25. https://doi.org/10.1080/ 16506073.2010.550058

22. Stein J, Röhr S, Luck T, Löbner M, Riedel-Heller S. Indikationen und Evidenz von international entwickelten Online-Coaches zur Intervention bei psychischen Erkrankungen - ein Meta-Review. Psychiatr Prax. 2018;45:7-15. https://doi.org/10.1055/s-0043-117050.

23. Chan Aea. SPIRIT 2013 explanation and elaboration: quidance for protocols of clinical trials. BMJ. 2013. https://doi.org/10.1136/bmj.e7586.

24. Ehlers A, Clark DM. A cognitive model of posttraumatic stress disorder. Behav Res Ther. 2000;38:319-45. https://doi.org/10.1016/S00057967(99)00123-0.

25. Benight CC, Ruzek Jl, Waldrep E. Internet interventions for traumatic stress: a review and theoretically based example. J Trauma Stress. 2008;21:513-20. https://doi.org/10.1002/jts.20371.

26. Steinmetz SE, Benight CC, Bishop SL, James LE. My disaster recovery: a pilot randomized controlled trial of an internet intervention. Anxiety Stress Coping. 2012;25:593-600. https://doi.org/10.1080/10615806.2011.604869.

27. American Psychiatric Association. Diagnostic and statistical manual of mental disorders. 5th ed. Washington, DC: Author; 2013.

28. Blevins CA, Weathers FW, Davis MT, Witte TK, Domino JL. The posttraumatic stress disorder checklist for DSM-5 (PCL-5): development and initial psychometric evaluation. J Trauma Stress. 2015;28:489-98. https://doi.org/10. 1002/jts.22059.

29. Faugier J, Sargeant M. Sampling hard to reach populations. J Adv Nurs. 1997;26:790-7. https://doi.org/10.1046/j.1365-2648.1997.00371.x.

30. Foa EB, McLean CP, Zang Y, Zhong J, Powers MB, Kauffman BY, et al. Psychometric properties of the posttraumatic diagnostic scale for DSM-5 
(PDS-5). Psychol Assess. 2016;28:1166-71. https://doi.org/10.1037/ pas0000258.

31. Kroenke K, Spitzer RL, Williams JBW. The PHQ-9. J Gen Intern Med. 2001;16 606-13. https://doi.org/10.1046/j.1525-1497.2001.016009606.x.

32. Joiner TE, Pfaff JJ, Acres JG. A brief screening tool for suicidal symptoms in adolescents and young adults in general health settings: reliability and validity data from the Australian national general practice youth suicide prevention project. Behav Res Ther. 2002;40:471-81. https://doi.org/10.1016/ S0005-7967(01)00017-1.

33. Harkness JA. Questionnaire translation. In: Harkness JA, van de Vijver F, Mohler PP, editors. Cross-cultural survey methods. Hoboken, NJ: John Wiley \& Sons; 2003. p. 35-56.

34. Harkness JA. Round 4 ESS translation strategies and procedures. Eur Soc Survey. 2008:1-14.

35. Haas H. Übersetzungsprobleme in der interkulturellen Befragung. Interculture J. 2009;8:61-77.

36. Brooke J. SUS-A quick and dirty usability scale. Usability Eval Ind. 1996;189: 4-7.

37. Venkatesh $\mathrm{V}$, Bala $\mathrm{H}$. Technology acceptance model 3 and a research agenda on interventions. Decis Sci. 2008;39:273-315. https://doi.org/10. 1111/j.1540-5915.2008.00192.x.

38. Knaevelsrud C, Brand J, Lange A, Ruwaard J, Wagner B. Web-based psychotherapy for posttraumatic stress disorder in war-traumatized Arab patients: randomized controlled trial. J Med Internet Res. 2015;17:e71. https://doi.org/10.2196/jmir.3582.

39. Faul F, Erdfelder E, Lang A-G, Buchner A. G*power 3: a flexible statistical power analysis program for the social, behavioral, and biomedical sciences. Behav Res Methods. 2007:39:175-91.

40. Snow G. Blockrand: randomization for block random clinical trials. 2013 [Accessed 2018].

41. R Core Team. R: a language and environment for statistical computing. Vienna, Austria: R Foundation for Statistical Computing; 2017.

42. van Buuren S. Flexible imputation of missing data. Boca Raton, 25-52: Chapman and Hall/CRC; 2012.

43. Bock J, Brettschneider C, Seidl H, Bowles D, Holle R, Greiner W, König HH. Ermittlung standardisierter Bewertungssätze aus gesellschaftlicher Perspektive für die gesundheitsökonomische Evaluation. Gesundheitswesen. 2015:77:53-61. https://doi.org/10.1055/s-0034-1374621.

44. Zethraeus N, Johannesson M, Jönsson B, Löthgren M, Tambour M. Pharmacoeconomics. 2003;21:39-48.

45. Moher D, Hopewell S, Schulz KF, Montori V, Gøtzsche PC, Devereaux PJ, et al. CONSORT 2010 explanation and elaboration: updated guidelines for reporting parallel group randomised trials. J Clin Epidemiol. 2010;63:e1-37. https://doi.org/10.1016/j.jclinepi.2010.03.004.

46. Rodriguez-Paras C, Tippey K, Brown E, Sasangohar F, Creech S, Kum H, et al. Posttraumatic stress disorder and Mobile health: app investigation and scoping literature review. JMIR Mhealth Uhealth. 2017;5:e156. https://doi. org/10.2196/mhealth.7318.

47. von Glischinski M, Teismann T, Prinz S, Gebauer JE, Hirschfeld G. Depressive symptom inventory suicidality subscale: optimal cut points for clinical and non-clinical samples. Clin Psychol Psychother. 2016;23:543-9. https://doi.org/ 10.1002/cpp.2007.

48. Spitzer RL, Kroenke K, Williams JBW. Validation and utility of a self-report version of PRIME-MD: The PHQ primary care study. JAMA. 1999;282:1737-44.

49. The EG. EuroQol_-A new facility for the measurement of health-related quality of life. Health Policy. 1990;16:199-208.

50. Schwarzer R, Jerusalem M. Skalen zur Erfassung von Lehrer- und Schülermerkmalen. Dokumentation der psychometrischen Verfahren im Rahmen der Wissenschaftlichen Begleitung des Modellversuchs Selbstwirksame Schulen. 1999. http://www.psyc.de/skalendoku.pdf.

51. Corrigan PW, Michaels PJ, Vega E, Gause M, Watson AC, Rüsch N. Selfstigma of mental illness scale--short form: reliability and validity. Psychiatry Res. 2012;199:65-9. https://doi.org/10.1016/j.psychres.2012.04.009.

52. Lubben JE. Assessing social networks among elderly populations. Fam Community Health. 1988:42-52.

53. Kendel F, Spaderna H, Sieverding M, Dunkel A, Lehmkuhl E, Hetzer R, RegitzZagrosek V. Eine deutsche adaptation des ENRICHD social support inventory (ESSI). Diagnostica. 2011;57:99-106. https://doi.org/10.1026/0012-1924/ a000030.

54. Tedeschi RG, Calhoun LG. The posttraumatic growth inventory: measuring the positive legacy of trauma. J Trauma Stress. 1996;9:455-71.
55. Leppert K, Koch B, Brähler E, Straß B. Die Resilienzskala (RS) - Überprüfung der Langform RS-25 und einer Kurzform RS-13. Klinische Diagnostik und Evaluation. 2008:226-43.

56. Huber S. Kerndimensionen, Zentralität und Inhalt. Ein interdisziplinäres Modell der Religiosität. J Psychol. 2008;16:1-17.

57. Lumbeck G, Brandstätter M, Geissner E. Erstvalidierung der deutschen version des "inventory of complicated grief" (ICG-D). Z Klin Psychol Psychother. 2012;41:243-8. https://doi.org/10.1026/1616-3443/a000172.

58. Leicht H, König H, Stuhldreher N, Bachmann C, Bickel H, Fuchs A, et al. Predictors of costs in dementia in a longitudinal perspective. PLoS One. 2013;8:e70018. https://doi.org/10.1371/journal.pone.0070018.

\section{Ready to submit your research? Choose BMC and benefit from:}

- fast, convenient online submission

- thorough peer review by experienced researchers in your field

- rapid publication on acceptance

- support for research data, including large and complex data types

- gold Open Access which fosters wider collaboration and increased citations

- maximum visibility for your research: over $100 \mathrm{M}$ website views per year

At BMC, research is always in progress.

Learn more biomedcentral.com/submissions 